\title{
The Origin of Follicular Bile Acids in the Human Ovary
}

Ruxandra A. Nagy, ${ }^{\dagger \dagger}$ Harry Hollema, ${ }^{\ddagger}$ Daniela Andrei, ${ }^{* \dagger}$ Angelika Jurdzinski, ${ }^{*}$ Folkert Kuipers, ${ }^{\star \S}$ Annemieke Hoek, ${ }^{\dagger}$ and Uwe J.F. Tietge ${ }^{* \mathbb{T} \|}$

From the Department of Pediatrics, * the Section of Reproductive Medicine, ${ }^{\dagger}$ Department of Obstetrics and Gynecology, the Department of Pathology, ${ }^{\ddagger}$ and the Department of Laboratory Medicine, ${ }^{\S}$ University of Groningen, University Medical Center Groningen, Groningen, the Netherlands; the Division of Clinical Chemistry, "Department of Laboratory Medicine, Karolinska Institutet, Stockholm, Sweden; and Clinical Chemistry, "Karolinska University Laboratory, Karolinska University Hospital, Stockholm, Sweden

Accepted for publication June 10, 2019.

Address correspondence to Uwe J.F. Tietge, M.D., Ph.D., Division of Clinical Chemistry, Department of Laboratory Medicine, H5, Alfred Nobels Alle 8, Karolinska Institutet, S14183 Stockholm, Sweden. Email: uwe.tietge@ki.se.

\begin{abstract}
Bile acids (BAs) are present in ovarian follicular fluid (FF) and are linked to embryo development. However, information on the source of ovarian BA is scarce. Therefore, we aimed to explore local ovarian synthesis and BA transport from blood into FF. BA levels were determined in matching FF and serum from women undergoing in vitro fertilization. In vitro BA production by human mural granulosa cells (MGCs) and cumulus granulosa cells (CGCs) was measured by mass spectrometry. Gene and protein expression were quantified in MGC and CGC and in human ovarian tissue by quantitative PCR and Western blot/immunohistochemistry, respectively. BA levels in blood and FF were significantly correlated $\left(r_{s}=0.186, P=0.027\right)$ but were almost twofold higher in $\mathrm{FF}(P<0.001)$. Primary BA levels were increased in $\mathrm{FF}$, indicating that, in addition to passive diffusion, other sources of ovarian $\mathrm{BA}$ might exist. The key BA synthesis enzyme cytochrome P450 A1 was absent in MGC and CGC; BA production in vitro was undetectable. Therefore, local ovarian BA production is unlikely. However, common BA importers $\left(\mathrm{Na}^{+} /\right.$taurocholate cotransporting polypeptide, apical sodium-dependent bile acid transporter) and an exporter (ATP-binding cassette subfamily C member 3 ) were identified in GC, theca cells, and oocyte. In summary, these results suggest that passive and active transport of BAs from blood into FF constitute sources of FF BA. (Am J Pathol 2019, 189: 2036-2045; https://doi.org/10.1016/ j.ajpath.2019.06.011)
\end{abstract}

Before ovulation, oocytes develop in ovarian follicles. Each preovulatory follicle has an external vascularized layer of theca cells and an avascular internal space that consists of granulosa cells (GCs), the oocyte, and follicular fluid (FF). ${ }^{1}$ GCs are metabolically active cells that line the interior of the follicle [mural GCs (MGCs)] and form a layer around the oocyte [cumulus GCs (CGCs)]. The proximity of the CGC to the oocyte is functionally important. ${ }^{2}$ Indeed, oocyte maturation is regulated by the surrounding $\mathrm{CGC}^{3}$ which provides most of its metabolic substrates. ${ }^{4,5}$ Moreover, the oocyte regulates expansion of the CGC population, which in turn has been shown to impact fertility. ${ }^{6}$ Thus, a functioning relationship between CGCs and the oocyte is crucial for oocyte development.

In the growing follicle, GCs contribute to the accumulation of increasing amounts of FF that surround the oocyte. FF thus constitutes the natural environment of the oocyte during maturation. Moreover, alterations in its composition have been linked to oocyte development and embryo quality. ${ }^{7-9}$ The composition of FF is determined by two main factors: passive or active transport of metabolites from the systemic circulation and local production by GCs. Passage of metabolites from blood into FF seems to be size-dependent, with

\footnotetext{
Supported by the Junior Scientific Masterclass of the University of Groningen (R.A.N.), departmental funds from the Department of Gynecology and Obstetrics (University Medical Center Groningen), and the Jan Kornelis de Cock Foundation (R.A.N. and U.J.F.T.). The Department of Obstetrics and Gynecology at the University Medical Center Groningen received an unrestricted educational grant from Ferring Pharmaceuticals BV (Hoofddorp, the Netherlands).

A.H. and U.J.F.T. contributed equally to this work as senior authors.

Disclosures: None declared.
} 
small components passing freely and larger ones selectively or facilitated by transporters, if at all. ${ }^{1}$ Therefore, alterations in the maternal blood composition of smaller molecules are expected to be to a certain extent reflected in FF, the microenvironment of the developing oocyte. ${ }^{10} \mathrm{With}$ regard to local production, GCs are capable of modifying the composition of FF by secreting or degrading metabolites. For example, in addition to local steroid production, ${ }^{11}$ human GCs were shown to secrete very-low-density lipoproteins, and higher levels of FF very-low-density lipoproteins were correlated with better in vitro fertilization (IVF) outcomes. ${ }^{12}$ Thus, the final FF composition of the mature follicle is a reflection of systemic maternal and local follicular metabolism.

Bile acids (BAs) are steroid compounds that are classically involved in fat and vitamin absorption in the gut, but increasingly are recognized for their endocrine roles, such as in glucose and lipid metabolism. ${ }^{13,14}$ BA production occurs via two pathways: the classic (neutral) and the alternative (acidic) pathway. ${ }^{15}$ To preserve the BA pool, loss of BA is prevented by active reuptake from the digestive tract into the blood stream via specialized transporters in the intestine and liver. ${ }^{16}$ Importantly, BAs have been shown to be present in FF and are linked to embryo development in IVF, indicating that BAs may have biological relevance for oocyte maturation. ${ }^{8}$ Hence, it is conceivable that alterations in the FF BA pool may have an impact on the oocyte and, consequently, on embryo development. However, the origin of BAs in FF has not been clarified. In addition to simple diffusion from blood into FF, other sources of BAs, such as local production and active transport, could play a role. ${ }^{17}$ The present study thus aimed to delineate the origin of ovarian BAs with a focus on the two possibilities of local intrafollicular synthesis and import from the blood compartment.

\section{Materials and Methods}

\section{FF and Serum Collection}

$\mathrm{FF}$ and serum from patients undergoing modified natural cycle (MNC) IVF at the University Medical Center Groningen (Groningen, the Netherlands) were collected during a multicenter cohort study on the outcomes of MNC-IVF. ${ }^{18}$ Our group previously reported on BA levels in a subset of these samples, consisting of only samples from the first IVF cycle of a patient. ${ }^{8,18}$ For the present study, samples from a subsequent IVF cycle were also used. Details on patient inclusion and study protocol have been published previously. ${ }^{8,18}$ A universal consent form was signed by all patients and their confidentiality was protected during the study by assigning patients and samples with untraceable codes. None of the patients objected to the use of their socalled waste material (such as FF, GCs, and surplus serum), which routinely becomes available during patient care and otherwise would be discarded. Fasted blood was drawn on the morning of oocyte retrieval and surplus serum was stored under the respective study code at $-20^{\circ} \mathrm{C}$. After oocyte collection, the remaining FF was centrifuged for 20 minutes at $300 \times g$ and the supernatant was stored under the respective study code at $-20^{\circ} \mathrm{C}$.

For the current study, FF and matching blood samples fulfilling the following criteria were used: FF was free of blood contamination upon visual inspection, no oocytes or only one oocyte was retrieved, and only one mature follicle was detected by ultrasound at ovum pickup.

Ethical approval from the Institutional Review Board was requested but waived because materials were anonymized, and patients had signed the universal consent form.

\section{MNC-IVF Procedure and Collection of FF for BA Analysis}

The MNC-IVF procedure has been described in detail previously. ${ }^{18,19}$ In contrast to classic hyperstimulation IVF, in which multiple dominant follicles develop in each cycle, in MNC-IVF only one dominant follicle develops and its contents are retrieved at ovum pickup. Consequently, the composition of the FF can be compared with that of the matching blood sample accurately.

In short, when the diameter of a natural growing ovarian follicle (measured by vaginal ultrasonography) reached 14 $\mathrm{mm}$, daily injections of $0.25 \mathrm{mg}$ gonadotropin-releasing hormone antagonist and 150 IU recombinant folliclestimulating hormone were started. When the diameter of the dominant follicle reached a minimum of $18 \mathrm{~mm}$ and/or serum estradiol levels exceeded $0.8 \mathrm{nmol} / \mathrm{L}, 10,000 \mathrm{IU}$ human chorionic gonadotropin was administered to prevent ovulation. Approximately 34 hours later the oocyte was retrieved with a single-lumen aspiration needle and without flushing of the follicle. The oocyte was inseminated following standard procedures. If macroscopic blood contamination was absent, the FF was centrifuged for 20 minutes at $300 \times g$ and the supernatant was stored at $-80^{\circ} \mathrm{C}$ for later analysis.

\section{IVF Procedure and Collection of GCs in Controlled Ovarian Hyperstimulation}

The controlled ovarian hyperstimulation IVF procedure has been described in detail elsewhere. ${ }^{20}$ In short, on day 23 of the previous menstrual cycle or during the use of oral contraceptives, patients were started on a hormonal downregulation protocol with daily subcutaneous triptorelin, 0.1 $\mathrm{mg}$ gonadotropin-releasing hormone analog, and 150 to 225 IU human menopausal gonadotropin up until the day of human chorionic gonadotropin injection. When the diameter of at least three dominant follicles reached a minimum of 18 $\mathrm{mm}, 5000$ IU human chorionic gonadotropin was administered for final maturation of the follicle and oocyte. Approximately 36 hours later, the oocytes were retrieved with a single-lumen aspiration needle. CGC aggregates were separated from the oocyte manually and used for experiments. After removal of the cumulus-oocyte complexes, 
the FF was saved in $50 \mathrm{~mL}$ Falcon tubes (Corning Life Sciences, Amsterdam, the Netherlands) at $37.5^{\circ} \mathrm{C}$ and $5 \%$ $\mathrm{CO}_{2}$ until they could be used for isolation of MGCs later the same day. Only the laboratory technician and the fertility physician who were involved in the clinical procedure were aware of the identity and characteristics of the patient, but not the researchers.

\section{CGC and MGC Isolation and Culture}

Primary MGCs and CGCs were obtained from preovulatory follicles from women undergoing controlled ovarian hyperstimulation IVF at the University Medical Center Groningen. CGCs were separated from the cumulus-oocyte complex manually and washed once in Hank's balanced salt solution (Thermo Fisher Scientific, Bleiswijk, the Netherlands) before being brought into culture or stored for further analysis. For the isolation of MGCs, FF was centrifuged for 5 minutes at $400 \times g$, followed immediately by 5 minutes at $500 \times g$. Red blood cells were removed by layering the cell pellet on a $40 \%$ Percoll solution (GE Healthcare, Uppsala, Sweden), followed by centrifugation for 10 minutes at $550 \times \mathrm{g}$. The suspension of GC was pipetted out gently and washed twice in Hank's balanced salt solution to remove the Percoll solution. Both CGCs and MGCs were stored for later analysis or brought into culture. In the latter case, both CGCs and MGCs first were incubated in trypsin for 3 minutes at $37^{\circ} \mathrm{C}$, followed by dispersion of potential clumps by passage through a $40-\mu \mathrm{m}$ cell strainer (Corning, Durham, NC).

Finally, primary CGCs and MGCs were plated in 12-well plates (Corning) at a density of 300,000 cells/well, and were left to attach for 2 days in basal medium [Dulbecco's modified Eagle medium/nutrient mixture F12 (Gibco, Paisley, UK) supplemented with $10 \%$ fetal calf serum (Lonza, Verviers, Belgium) and 1\% penicillin/streptomycin/ amphotericin B (Lonza, Walkersville, MA)] at $37^{\circ} \mathrm{C}$ and $5 \%$ $\mathrm{CO}_{2}$. The medium was refreshed after 2 days and then daily until the culture was devoid of red blood cells on visual inspection (mean $=4$ days). Thereafter, the cells were cultured for 63 hours in medium with either $0.34 \mathrm{mmol} / \mathrm{L}$ low-density lipoprotein cholesterol, $0.34 \mathrm{mmol} / \mathrm{L}$ highdensity lipoprotein cholesterol, or the equivalent volume of phosphate-buffered saline (Gibco). Fetal calf serum was not added to this medium because it contains BA. Lowdensity and high-density lipoproteins were isolated by sequential ultracentrifugation $(1.019<\mathrm{d}<1.063$ and $1.063<\mathrm{d}<1.21$, respectively) from plasma of healthy male volunteers. HepG2 cells (a hepatoma cell line) and empty wells were used as positive and negative controls for each condition, respectively.

\section{BA Measurements}

In blood and FF, measurement of total BA was conducted using an enzymatic fluorimetric assay and by liquid chromatography-mass spectrometry as previously described. ${ }^{8}$ In cell culture supernatants and cell lysates, BAs were measured by liquid chromatography-mass spectrometry as previously described. ${ }^{8}$

\section{Protein Measurement}

Total protein levels were measured in matched FF and serum using the BCA Protein Assay Kit (Pierce, Rockford, IL) following the manufacturer's instructions.

\section{Isolation of RNA and Measurement of mRNA Levels by Real-Time Quantitative PCR}

Total RNA was obtained from freshly isolated MGCs and CGCs and from healthy human livers using TRI Reagent (Sigma, St. Louis, MO) and quantified with a Nanodrop spectrophotometer (Nanodrop 2000c; Thermo Fisher Scientific). Complementary DNA was synthesized from $1 \mu \mathrm{g}$ of RNA using Moloney-Murine Leukemia Virus Reverse Transcriptase (Thermo Fisher Scientific). Real-time quantitative PCR analysis was performed on a real-time PCR system (StepOnePlus, Applied Biosystems, Thermo Fisher Scientific). Primers and fluorogenic probes (Table 1) were designed with the Primer Express software version 2.0 (Thermo Fisher Scientific) and synthesized by Eurogentec (Seraing, Belgium). Gene expression levels were normalized to the housekeeping gene peptidylprolyl isomerase $\mathrm{G}$, and calculated using the $\Delta C_{T}$ method.

\section{Western Blot Analysis}

Western blot analyses for $\mathrm{Na}^{+} /$taurocholate cotransporting polypeptide (NTCP), apical sodium-dependent bile acid transporter (ASBT), ATP-binding cassette subfamily C member 4 (ABCC4) and $\mathrm{ABCC} 3$ were performed on primary human MGCs and CGCs pooled from several patients. Protein was resolved by SDS-PAGE and subsequently blotted onto nitrocellulose (Trans-Blot Turbo Transfer Pack; Bio-Rad, Hercules, CA). The proteins were visualized using the NTCP antibody (kindly provided by Professor Dr. Bruno Stieger, University Hospital Zurich, Zurich, Switzerland; raised in rabbit; dilution 1:500), ASBT antibody (kindly provided by Professor Paul A. Dawson, Wake Forest University School of Medicine, Winston-Salem, NC; raised in rabbit; dilution 1:10,000), commercially available ABCC4 antibody (M4I-10, NBP1-42339; Novus Biologicals, Minneapolis, MN; raised in rat; dilution 1:200), and commercially available ABCC3 antibody (sc-5776, C18; Santa Cruz Biotechnology, Santa Cruz, CA; raised in goat; dilution 1:500), followed by the appropriate horseradish-peroxidase-conjugated secondary antibody [goat anti-rabbit (P0448; Dako, Heverlee, Belgium), rabbit antigoat (P0449; Dako), and goat anti-rat (HAF005; R\&D Systems, Minneapolis, MN)]. 
Table 1 Primer Sequences

\begin{tabular}{|c|c|}
\hline Gene name & Sequence \\
\hline \multirow[t]{3}{*}{ PPIG } & F: 5'-TGGAGCCATGGGAATAAAGGT-3' \\
\hline & R: 5'-CTCTTCCAGCAGGTTGATTGTTAAT-3' \\
\hline & $\begin{array}{l}\text { P: 5'-CAACGTCCTCGATGTTTTTTTGACAT- } \\
\text { TGCC-3' }\end{array}$ \\
\hline \multirow[t]{3}{*}{ CYP7A1 } & F: 5'-TCAGCTTGGAAGGCAATCCTAT-3' \\
\hline & R: 5'-AGCCTCAGCGATTCCTTGATTA-3' \\
\hline & P: 5'-CTGGCAGGTCATTCAGTTCTGCTTGACTC-3' \\
\hline \multirow[t]{3}{*}{ CYP8B1 } & F: 5'-CCTGAGCTTGTTCGGCTACAC-3' \\
\hline & R: 5'-TGCGGAACTCCATGAATAACTCTC-3' \\
\hline & P: 5'-CCTGTAGCAGGTCCTGCTCCTTGTCCTT-3' \\
\hline \multirow[t]{3}{*}{ CYP27A1 } & F: 5'-TGCGGGCAGAGAGTGCTT-3' \\
\hline & R: 5'-ACAGGATGTAGCAAATAGCTTCCA-3' \\
\hline & P: 5'-CAGGTGTCGGACATGGCTCAACTCTTCT-3' \\
\hline \multirow[t]{3}{*}{ CYP7B1 } & F: 5'-CTTGAAATAGGAGCACATCATTTAGG-3' \\
\hline & R: 5'-GATAATACATTGCCCAGAACATAGTTG-3' \\
\hline & P: 5'-CTCTGGGCCTCTGTGGCAAACACTATTC-3' \\
\hline \multirow[t]{3}{*}{ FXR } & F: 5'-AGGGGTGTAAAGGTTTCTTCAGGA-3' \\
\hline & R: 5'-ACACTTTCTTCGCATGTACATATCCAT-3' \\
\hline & P: 5'-TTGCCCCCGTTTTTACACTTGTACACAGC-3' \\
\hline \multirow[t]{3}{*}{$R X R-\alpha$} & F: 5'-GCAAACATGGGGCTGAACC-3' \\
\hline & R: 5'-GCTGCTTGGCAAATGTTGGT-3' \\
\hline & P: 5'-CAGCTCGCCGAACGACCCTGTC-3' \\
\hline \multirow[t]{3}{*}{$L X R-\alpha$} & F: 5'-CTTGCTCATTGCTATCAGCATCTT-3' \\
\hline & R: 5'-ACATATGTGTGCTGCAGCCTCT-3' \\
\hline & P: 5'-TCTGCAGACCGGCCCAACGTG-3' \\
\hline \multirow[t]{3}{*}{ LRH1 } & F: 5'-CAGAGAACTTAAGGTTGATGACCAA-3' \\
\hline & R: 5'-GGTAAATGTGGTCGAGGATTAAGAG-3' \\
\hline & P: 5'-TCACTCCAGCAGTTCTGAAGCAGCTTCA-3' \\
\hline \multirow[t]{3}{*}{ TGR5 } & F: 5'-CGTCTACTTGGCTCCCAACTTC-3' \\
\hline & R: 5'-GGCCTCAGGACTGCCATGTA-3' \\
\hline & P: 5'-СTCTCCCTGCTTGCCAACCTCTTGC-3' \\
\hline \multirow[t]{3}{*}{$V D R$} & F: 5'-CCGCATCACCAAGGACAAC- $3^{\prime}$ \\
\hline & R: 5'-TCATCTGTCAGAATGAACTCCTTCA-3' \\
\hline & P: $5^{\prime}-$ AGGCCTGCCGGCTCAAACGC- $3^{\prime}$ \\
\hline \multirow[t]{3}{*}{ PXR } & F: 5'-ACATGCTGAAGAAGCTGCAGCT- $3^{\prime}$ \\
\hline & R: 5'-GGCGGTCTGGGGAGAAGA-3' \\
\hline & P: 5'-ATGGCCTGCATCAGCACATACTCCTCC-3' \\
\hline \multirow[t]{3}{*}{ CAR } & F: 5'-ACCGACCTGGAGTTACCCAGA-3' \\
\hline & R: 5'-CTTCGCATACAGAAACCGATCC-3' \\
\hline & P: 5'-CTTTGCAGAGTCAGTGCCATCTCCTCTTG-3' \\
\hline \multirow[t]{3}{*}{ FGFR4 } & F: 5'-TGTGCAAGGTGTACAGCGATG-3' \\
\hline & R: 5'-TATTGATGTCTGCAGTCTTTAGGACTT-3' \\
\hline & P: 5'-CGTCATCAACGGCAGCAGCTTCG-3' \\
\hline \multirow[t]{3}{*}{ FGF19 } & F: 5'-ATGCAGGGGCTGCTTCAGTA-3' \\
\hline & R: $5^{\prime}-$ AGCCATCTGGGCGGATCT-3' \\
\hline & P: 5'-TCCTCGAAAGCACAGTCTTCCTCCG-3' \\
\hline \multirow[t]{3}{*}{$K L B$} & F: 5'-AATGGCTGGTTCACAGACAGTC-3' \\
\hline & R: 5'-TCATCTAАССTTATTGCTTGAAGCA-3' \\
\hline & $\begin{array}{l}\text { P: 5'-ACCACGGCCATCTACATGATGAAGAA- } \\
\text { TTTC- - }{ }^{\prime}\end{array}$ \\
\hline \multirow[t]{3}{*}{ NTCP } & F: 5'-TGATATCACTGGTCCTGGTTCTCA-3' \\
\hline & R: 5'-GCATGTATTGTGGCCGTTTG-3' \\
\hline & P: 5'-TCCTTGCACCATAGGGATCGTCCTCA-3' \\
\hline \multirow[t]{3}{*}{$A S B T$} & F: 5'-CACGCAGCTATGTTCCACCAT-3' \\
\hline & R: 5'-GAGCGGGAAGGTGAATACGA-3' \\
\hline & P: 5'-CAGCTCTCСТTCAСTCCTGAGGAGCTCA-3' \\
\hline
\end{tabular}

(table continues)
Table 1 (continued)

\begin{tabular}{|c|c|}
\hline Gene name & Sequence \\
\hline \multirow[t]{3}{*}{ OST- $\alpha$} & F: 5'-GGTGAGCAGAACATGGGAGC-3' \\
\hline & R: 5'-ATGGAGGGCTGTAGGGCAGT-3' \\
\hline & $\begin{array}{l}\text { P: 5'-AAATTTGCTCTGTTCCAGGTTCTCCTCA- } \\
\text { TCC-3' }\end{array}$ \\
\hline \multirow[t]{3}{*}{ OST- $\beta$} & F: 5'-CAGGAGCTGCTGGAAGAGAT-3' \\
\hline & R: 5'-GACCATGCTTATAATGACCACCA-3' \\
\hline & $\begin{array}{l}\text { P: 5'-CGTGTGGAAGATGCATCTCCCTGGAATCA- } \\
\text { TTC-3' }\end{array}$ \\
\hline \multirow[t]{3}{*}{$A B C B 11$} & F: 5'-ACATGCTTGCGAGGACCTTTA-3' \\
\hline & R: 5'-GGAGGTTCGTGCACCAGGTA-3' \\
\hline & P: 5'-CCATCCGGCAACGCTCCAAGTCT-3' \\
\hline \multirow[t]{3}{*}{$A B C C 3$} & F: 5'-GCCATCGACCTGGAGACTGA-3' \\
\hline & R: 5'-GACCCTGGTGTAGTCCATGATAGTG-3' \\
\hline & P: 5'-CATCCGCACCCAGTTTGATACCTGCAC-3' \\
\hline \multirow[t]{3}{*}{$A B C C 4$} & F: 5'-AAGTGAACAACCTCCAGTTCCAG- $3^{\prime}$ \\
\hline & R: 5'-GGCTCTCCAGAGCACCATCT-3' \\
\hline & $\begin{array}{l}\text { P: } 5^{\prime}-\text { CAAACCGAAGACTCTGAGAAGGTACGATT- } \\
\text { CCT-3' }\end{array}$ \\
\hline \multirow[t]{3}{*}{ OATP1B1 } & F: 5'-AAGCCACTTCTGCTTCTGTGTTT-3' \\
\hline & R: 5'-AATTCTTAGTGAAAGGACCAGGAACT-3' \\
\hline & 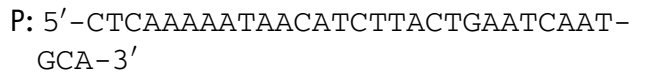 \\
\hline \multirow[t]{3}{*}{ OATP1B3 } & F: 5'-AACATGTAATTTGGACATGCAAGAC-3' \\
\hline & R: 5'-TTGTCAGTGAAAGACCAGGAACA-3' \\
\hline & $\begin{array}{l}\text { P: 5'-CTGCTGCCAACTAACATTGCATTGATTC- } \\
\text { АTT-3' }\end{array}$ \\
\hline
\end{tabular}

$A B C B$, ATP binding cassette subfamily $B$ member; $A B C C$, ATP-binding cassette subfamily $C$ member; $A S B T$, apical sodium-dependent bile acid transporter; $\mathrm{CAR}$, constitutive androstane receptor; CYP, cytochrome $\mathrm{P} 450$; $F$, forward; FGF, fibroblast growth factor; FXR, farnesoid $X$ receptor; KLB, klotho $\beta$; LRH1, liver receptor homolog 1; LXR, liver $X$ receptor; NTCP, $\mathrm{Na}^{+}$/ taurocholate cotransporting polypeptide; OATP, organic anion transporting polypeptide; OST, organic solute transporter; $\mathrm{P}$, probe; PPIG, peptidylprolyl isomerase $G$; PXR, påregnane $X$ receptor; $R$, reverse; $R X R$, retinoic acid receptor; TGR, Takeda G-protein-coupled receptor; VDR, vitamin D receptor.

\section{Immunohistochemistry}

Anonymized ovarian tissue from carriers of the BRCA mutation who underwent preventive oophorectomy was used. The tissue was fixed in formalin, embedded in paraffin, and sectioned into $3-\mu \mathrm{m}$ slides. Before staining, paraffin was removed by immersion of the slides in xylol solution, followed by washing in ethanol. For antigen retrieval, either magnesium citrate (ASBT and ABCC4), EDTA (NTCP), or Tris/EDTA buffer (ABCC3) was used. To reduce endogenous peroxidase activity, the slides were incubated in hydrogen peroxide solution for 30 minutes. The slides then were incubated for 1 hour at room temperature in primary antibody dissolved in $1 \%$ bovine serum albumin/phosphate-buffered saline. The following dilutions were used: 1:500 NTCP, 1:200 ASBT, 1:100 ABCC4 (Western Blot Analysis), and 1:100 ABCC3 (sc-5774, H-16; Santa Cruz Biotechnology; raised in goat). Thereafter, they were incubated with secondary and tertiary goat anti-rabbit antibodies (P0448; Dako), rabbit anti-goat (P0449; Dako), 
and rabbit anti-goat antibodies (P0450; Dako) diluted 1:100 in $1 \%$ bovine serum albumin with $1 \%$ corresponding serum solution prepared in phosphate-buffered saline. The order of these antibodies was determined by the nature of the primary antibody. The slides then were incubated for 10 minutes in 3,3'-diaminobenzidine and counterstained with hematoxylin. Finally, the slides were dehydrated and mounted.

\section{Statistical Analysis}

Results of the measurements of BAs in FF and matched serum and of measurements of total protein in FF and serum were expressed as the median (interquartile range). Their correlations were expressed as Spearman r. For BA measurements in matched FF and serum, the levels were compared statistically, using multilevel generalized estimating equations and the results were presented as odds ratio $(95 \% \mathrm{CI})$. For protein measurement in matched $\mathrm{FF}$ and serum, the levels were compared statistically using the Wilcoxon signed-rank test. Gene expression levels in CGCs and MGCs were compared statistically using the independent samples $t$-test if the values were distributed normally or the $U$-test if the values were not distributed normally. A $P$ value less than 0.05 was considered statistically significant. Analyses were conducted using SPSS version 23 (SPSS, Inc., Chicago, IL).

\section{Results}

\section{Passive Diffusion of BAs from Blood into FF}

Total BA levels were measured in FF and matching serum from $142 \mathrm{MNC}-\mathrm{IVF}$ procedures corresponding to 131 unique patients. A summary of cycle characteristics can be found in Supplemental Table S1. There was a weak positive correlation between total BAs in FF and in blood $\left(\mathrm{r}_{\mathrm{s}}=0.186 ; P=0.027\right.$ ) (Figure 1), and secondary BAs were present in FF (deoxycholic acid derivatives, 0.72 $\mu \mathrm{mol} / \mathrm{L}$; interquartile range, $0.39-1.19 \mu \mathrm{mol} / \mathrm{L} ; n=139$ ). Nonetheless, total BA levels were consistently approximately two-fold higher in FF $(10.10 \mu \mathrm{mol} / \mathrm{L}$; interquartile range, $8.38-11.93 \mu \mathrm{mol} / \mathrm{L}$ ) compared with matching serum samples $(5.89 \mu \mathrm{mol} / \mathrm{L}$; interquartile range, $4.15-7.88 \mu \mathrm{mol} /$ $\mathrm{L}$, odds ratio, 58.01; 95\% CI, 31.05-108.40; $P<0.001$ ) (Figure 2), and primary BAs were more abundant in FF (mean $=73 \%$ of total BAs) than in matched serum (mean $=60 \%$ of total BAs; $P<0.001$ ).

To study whether the higher levels of FF BAs may be related to a different protein composition of $\mathrm{FF}$ as compared with serum, total protein levels were measured in FF and matched serum samples from 10 individual patients for which total BA measurements were available. The levels of protein in FF were significantly lower than those in matched serum $(56.16 \mathrm{mg} / \mathrm{mL}$; interquartile range, $51.86-59.25 \mathrm{mg} / \mathrm{mL}$ versus $69.83 \mathrm{mg} / \mathrm{mL}$; interquartile

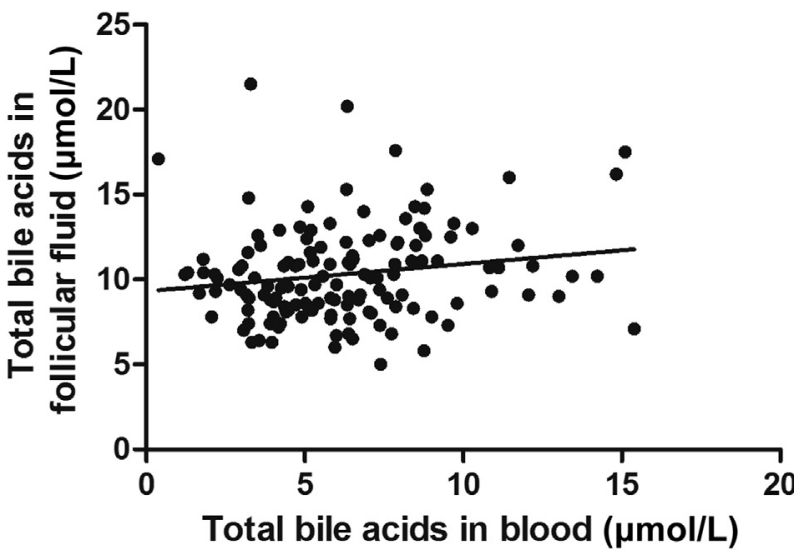

Figure 1 Relationship of total bile acid (BA) levels in follicular fluid and in matching blood. Black line represents the best fitting line for the relationship between total BAs in blood and in follicular fluid. $r_{\mathrm{s}}=0.186$. $P=0.027$

range, $67.54-76.32 \mathrm{mg} / \mathrm{mL} ; P=0.005$ ). Moreover, there was no correlation between protein levels and total BAs in $\mathrm{FF}\left(\mathrm{r}_{\mathrm{s}}=-0.006 ; P=0.987\right)$.

\section{Local Ovarian BA Production}

To study whether BAs are produced locally in the ovaries, mRNA expression of key genes involved in BA production and metabolism was measured in freshly isolated MGCs (nine individual patients) and CGCs (eight individual patients) (Figure 3). For the BA production enzymes, healthy human livers served as a positive control $(n=4)$. Before all analyses on primary MGCs and CGCs, the presence of anti-Muellerian hormone type-2 receptor (MISR-II; specific markers for GCs) and steroidogenic activity (ie, estrogen production upon induction with follicle-stimulating hormone and androstenedione) of the isolated primary cells was confirmed (data not shown). Gene expression of cytochrome P450 (CYP)7Al could not be detected in either

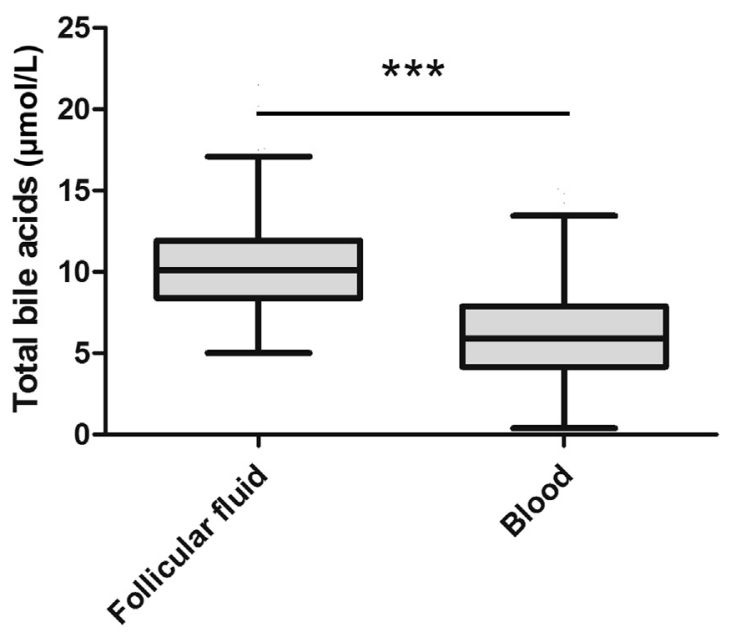

Figure 2 Comparison of total bile acid levels in follicular fluid and in matching blood. ${ }^{* *} P<0.001$. 

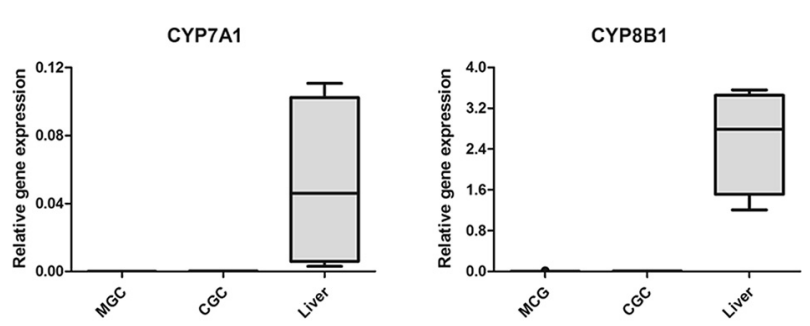

CYP27A1
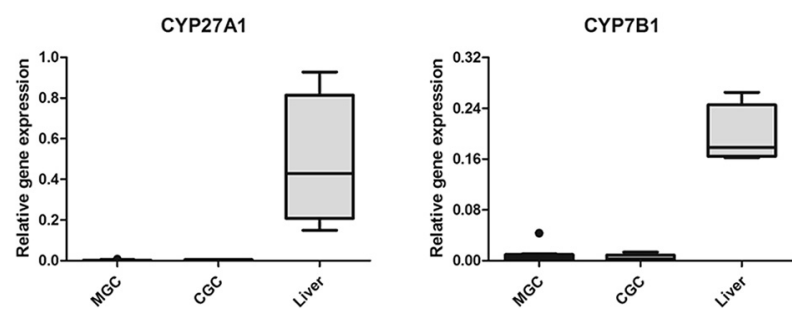

Figure 3 mRNA expression of key enzymes for bile acid production in mural granulosa cells (MGCs) and cumulus granulosa cells (CGCs) and human livers.

MGCs or CGCs, indicating that the classic pathway of BA production was not present. Very-low-level mRNA expression of CYP8B1, CYP27A1, and CYP7B1 was detected inconsistently in MGCs and CGCs, also making it highly unlikely that there is a discernible, substantial $d e$ novo synthesis of BAs via the alternative pathway in the ovary. Nonetheless, the nuclear receptors farnesoid $\mathrm{X}$ receptor, retinoic acid receptor- $\alpha$, liver $\mathrm{X}$ receptor- $\alpha$, and liver receptor homolog 1, as well as the G-protein-coupled bile acid receptor TGR5 were present in both cell types (Figure 4 and Supplemental Table S2). The level of mRNA expression of farnesoid $\mathrm{X}$ receptor, retinoic acid receptor- $\alpha$, liver $\mathrm{X}$ receptor- $\alpha$, and liver receptor homolog 1 was significantly higher in CGCs than in MGCs $(P=0.023$ for farnesoid $\mathrm{X}$ receptor, $P<0.001$ for all others).
To further formally test the concept of de novo synthesis of BAs via the alternative pathway in the ovary, MGCs and CGCs were cultured in medium without or with added lipoproteins as a substrate for BA synthesis $(0.34 \mathrm{mmol} / \mathrm{L}$ low-density lipoprotein or $0.34 \mathrm{mmol} / \mathrm{L}$ high-density lipoprotein). The BA content of cell supernatants and lysates was measured after 63 hours. In more than 10 individual experimental repeats, BAs could not be detected in any appreciable amounts in GC cultures (medium and cells), but consistently were present, although in low amounts, in material from HepG2 cultures.

\section{Active Transport of BAs from Blood into FF}

To explore the possibility of active transport of BAs from blood into FF, mRNA expression studies of common BA importer [NTCP, ASBT, organic anion transporting polypeptide (OATP)1B1, OATP1B3] and exporter [organic solute transporter subunit $\alpha$ (OST- $\alpha)$, OST- $\beta$, ATP binding cassette subfamily $B$ member 11 (ABCB11), ABCC3, ABCC4] proteins were performed in freshly isolated GCs, harvested from FF from controlled ovarian hyperstimulation IVF procedures. NTCP, ASBT, OST- $\alpha, A B C C 3$, and $A B C C 4$ transcripts were present at varying levels in MGCs and CGCs (Figure 5 and Supplemental Table S2). The level of gene expression of $A B C C 3$ was significantly higher in CGCs compared with MGCs $(P<0.001)$. For NTCP, ASBT, OST- $\alpha$, and $A B C C 4$ there was no significant difference in the levels of gene expression between the two GC types. OST- $\beta$ and ATP binding cassette subfamily B member 11 expression were below detection level in both cell types (data not shown). Because OST- $\alpha$ is functional only in the presence of OST- $\beta,{ }^{21}$ protein expression of OST- $\beta$ was not studied.
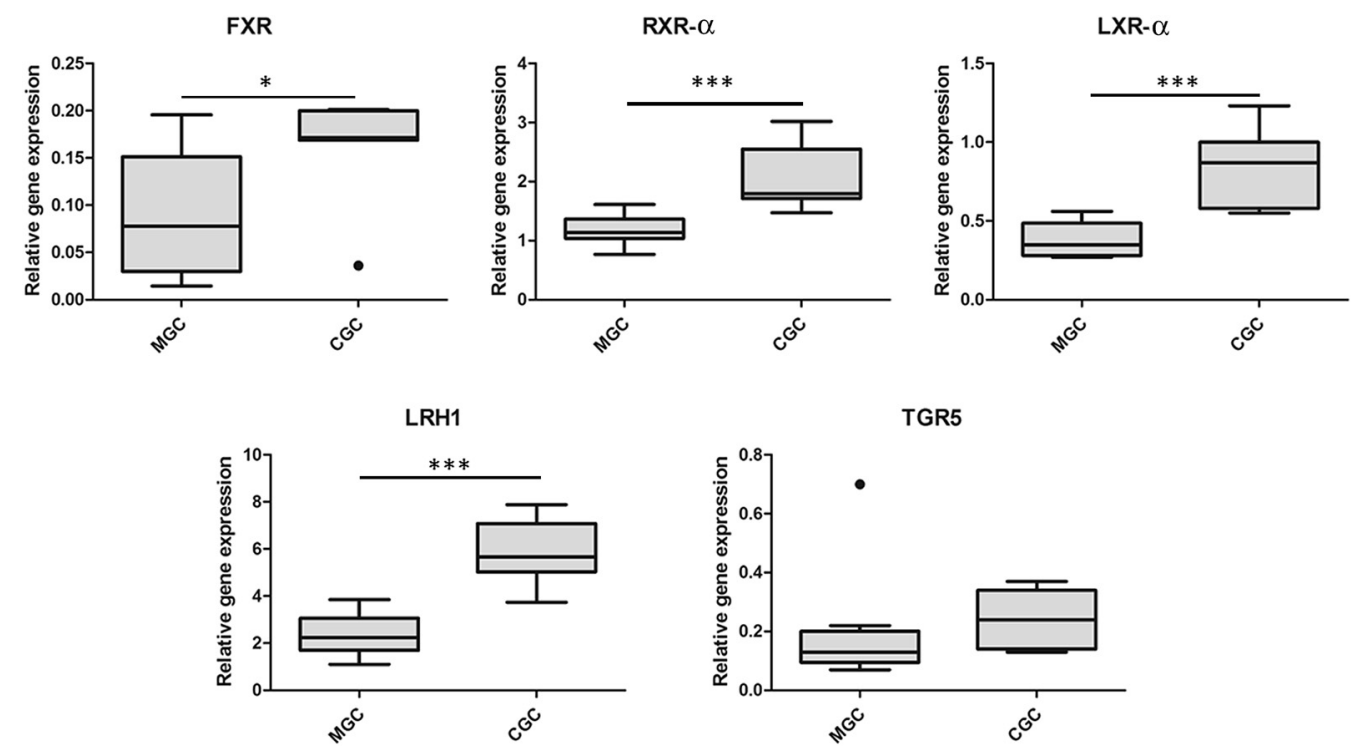

Figure 4 mRNA expression of bile acid-responsive receptors in mural granulosa cells (MGCs) and cumulus granulosa cells (CGCs). ${ }^{*} P<0.05$, ${ }^{* * *} P<0.001$. FXR, farnesoid $X$ receptor; LRH1, liver receptor homolog 1; LXR, liver X receptor; RXR, retinoic acid receptor. 
NTCP

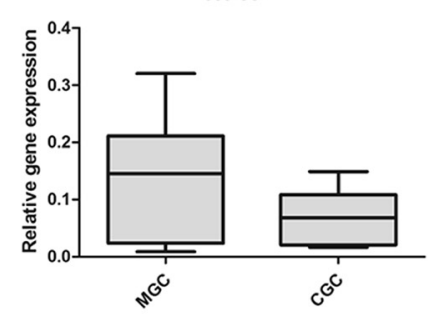

ASBT

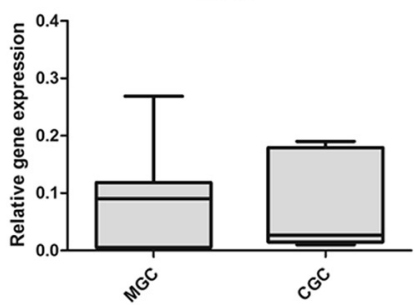

OST- $\alpha$

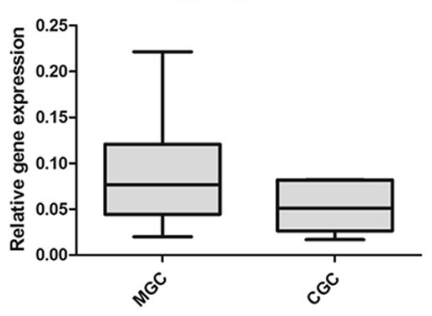

ABCC3

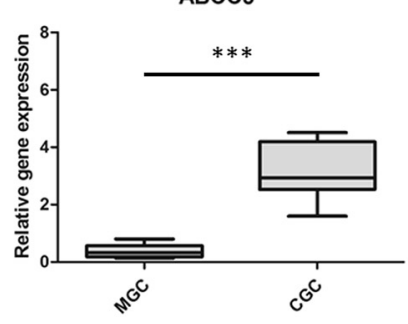

ABCC4

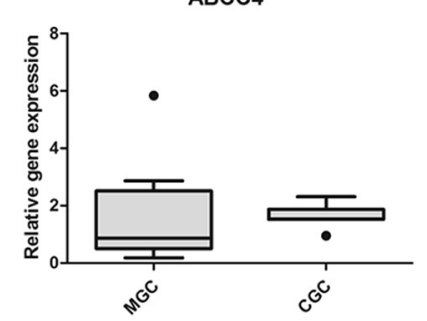

Figure 5 mRNA expression of common transport proteins involved in the import (top row) and export of bile acids (bottom row). ${ }^{* *} P<0.001$. ABCC, ATP-binding cassette subfamily C member; ASBT, apical sodium-dependent bile acid transporter; CGC, cumulus granulosa cell; MGC, mural granulosa cell; NTCP, $\mathrm{Na}^{+}$/taurocholate cotransporting polypeptide; OST, organic solute transporter subunit.

Next, protein expression of NTCP, ASBT, and ABCC3 was studied (Figure 6). Freshly isolated MGCs and CGCs from multiple patients were pooled and protein expression was assessed by Western blot. Immunohistochemistry was performed on paraffin-embedded human ovarian tissue. In tertiary follicles, the BA importers ASBT and NTCP were present in both theca and MGCs, and NTCP additionally was detected in CGCs. The organic anion exporter ABCC3 was present in theca cells, MGCs, and CGCs. ABCC3 expression could not be confirmed by Western blot of MGCs. The presence of the BA exporter ABCC4 was confirmed in Western blots of CGCs and MGCs, but not in immunohistochemical staining of ovarian tissue. Finally, in immunohistochemistry of primary and primordial follicles, NTCP and ABCC3 were present in GCs, whereas ASBT was absent. Finally, all three transporters also clearly were detectable in the oocyte.

\section{Discussion}

The results of the present study suggest that BAs present in FF in higher amounts compared with serum likely reach the FF from the systemic circulation by passive diffusion and active transport. Conversely, in human ovarian GCs, local BA production is highly unlikely to occur. To our knowledge, this is the first report of BA transporters being present in ovarian follicles.

The BA synthesis pathway traditionally is thought to be present exclusively in the liver. However, a previous microarray study in human GCs from hyperstimulation IVF suggested that components of the classic and alternative pathways of BA synthesis are present in human CGCs. ${ }^{17}$ In the current study, primary BAs were two-fold more abundant in FF than in serum, indicating that local production may take place. Moreover, in partial agreement with previous work, ${ }^{17}$ mRNA expression of certain BA synthesis enzymes of the alternative pathway (namely CYP27Al and $C Y P 7 B 1$ ) was found, although the expression was very weak and inconsistent. However, physiological relevance could not be confirmed because GCs did not produce BAs in any of the applied conditions. In addition, mRNA expression of CYP7A1, the key enzyme of the classic pathway, was completely absent. Combined, these data make it highly unlikely that BAs are being produced locally in the ovary. The cause of this discrepancy between previous results and ours is unclear, but it may be related to differences in culture conditions. ${ }^{17}$ In the present study, fetal calf serum was not added to the culture medium because this contains BAs and thus may bias the results.

Instead of local synthesis, our results suggest that ovarian BAs are being transported passively and actively into FF. To enter the FF, blood components must pass through the blood-follicular barrier composed of, from the exterior to the follicle, a vascular wall (endothelium and basement membrane), theca cells, follicular basement membrane, and GCs. ${ }^{1}$ Passage of molecules from blood into FF is size- and charge-dependent. ${ }^{1}$ BAs are small compounds that predominantly are present in blood bound to albumin and lipoproteins. ${ }^{22}$ With regard to the former, immunohistochemical studies have shown albumin to be present in the structures composing the blood-follicular barrier and the follicular antrum, suggesting that it passes unhindered from blood into FF. ${ }^{10}$ As for the latter means of transport, high-density lipoproteins are the sole type of lipoproteins present in FF. ${ }^{23}$ Given their small size, they are assumed to originate from blood by diffusion across the blood-follicular barrier. ${ }^{24}$ Indeed, there 
NTCP

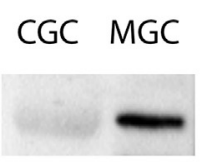

ASBT

CGC MGC
$\mathrm{ABCC} 3$

\section{CGC MGC}

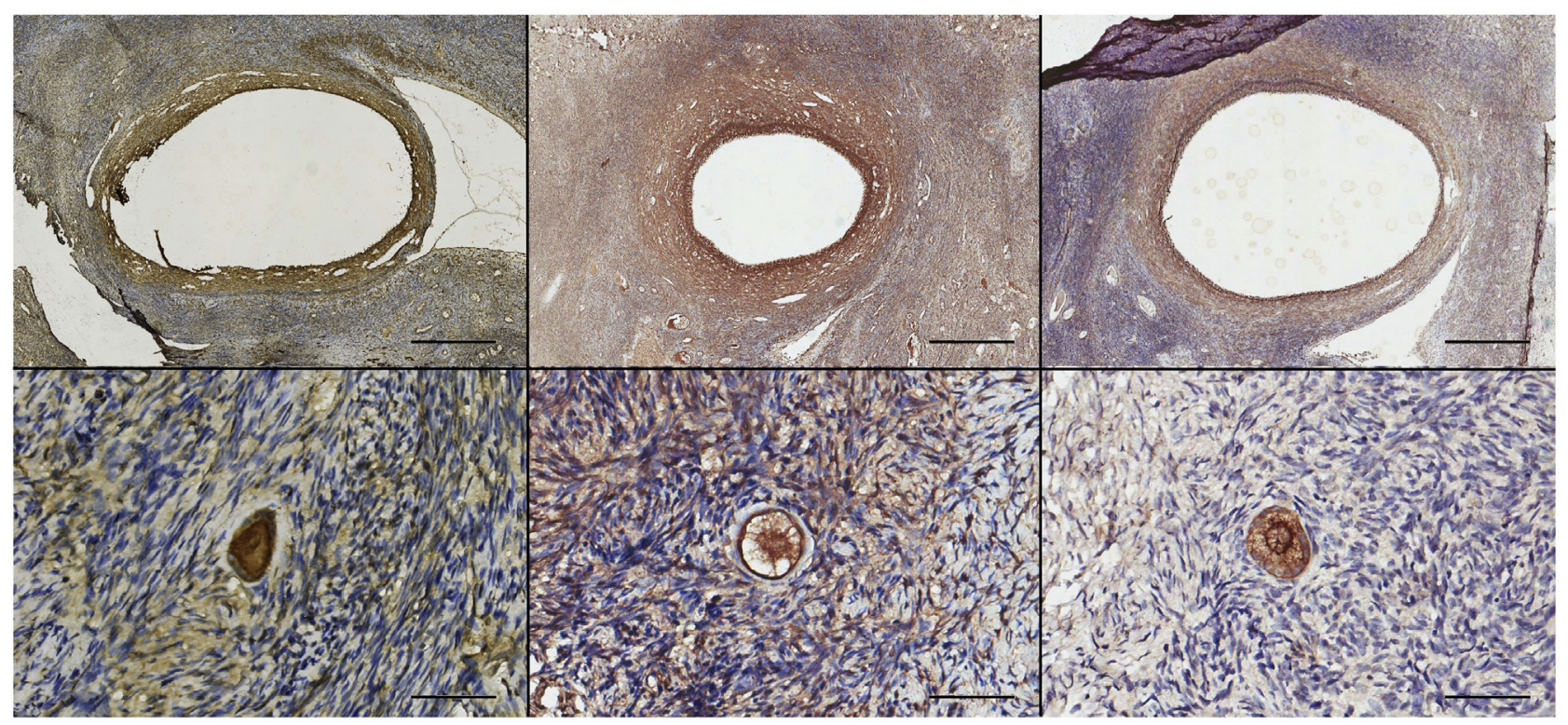

Figure 6 Protein expression of the bile acid importers $\mathrm{Na}^{+} /$taurocholate cotransporting polypeptide (NTCP) and apical sodium-dependent bile acid transporter (ASBT), and the bile acid exporter ATP-binding cassette subfamily C member 3 (ABCC3) in cell lysates of human primary cumulus granulosa cells (CGCS) and mural granulosa cells (MGCs) (top row: Western blot), and in ovarian tissue (middle row: immunohistochemical staining of tertiary follicles; bottom row: immunohistochemical staining of primordial and primary follicles). Scale bars: $500 \mu \mathrm{m}$ (middle row); $50 \mu \mathrm{m}$ (bottom row).

was a positive correlation between BAs in FF and those in blood, supporting the concept of passive diffusion. This was reinforced further by the presence of secondary BAs in FF, because secondary BAs originate from the modification by intestinal bacteria, which are not expected to be present in FF. In conclusion, BAs are likely to pass unhindered through the blood-follicular barrier as cargo of serum proteins, such as albumin, and possibly also high-density lipoproteins. However, the much higher levels of FF BAs as compared with blood (which is in agreement with the work of Smith et $\mathrm{al}^{17}$ ) and the lack of a correlation between protein levels and BA levels in FF already suggest that facilitated transport, in addition to diffusion, takes place. The relative overabundance of BA importers (NTCP and ASBT) over exporters (ABCC3) found in the present study may explain BA accumulation in FF. In the present study, we have focused on the most common BA transporters and it cannot be excluded that other, less well-known importers and exporters also may be present in ovarian follicles. Because active transport is not formally shown in the current study, studies quantifying the relative transport capacities of each transporter in and out of the FF would be interesting and additive to the current work. However, these are technically very challenging to perform and virtually impossible to perform in humans. Finally, it should be mentioned that blood BA concentrations show diurnal variations (ie, are higher after ingestion of a meal). Because blood was collected under fasting conditions, high FF BA concentrations may in part reflect an earlier exposure to high serum BA concentrations.

Accumulation of BAs in FF during oocyte development and the presence of BA transporters in the oocyte as well as of a signaling system responsive to BAs in the follicular environment argue in favor of a biological function of ovarian BAs in human reproduction. Over the past decade there has been extensive research indicating that BAs are more than just mere detergents, and also fulfill important endocrine functions. Indeed, they are involved in the regulation of glucose and lipid metabolism, immunity, and gut microbiota function via nuclear receptors such as farnesoid $\mathrm{X}$ receptor and TGR5. ${ }^{13}$ Moreover, BAs seem to be involved in fertility and programing of offspring health. Ursodeoxycholic acid derivatives in human FF are associated with the development of top-quality embryos. ${ }^{8}$ In animals, tauroursodeoxycholic acid has been shown to reduce apoptosis of mouse and pig embryos and to increase the implantation and live birth rate in mice. ${ }^{25-27}$ In male rodents, supplementation of the diet with cholic acid results in reduced fertility, altered sperm methylation patterns, and increased perinatal mortality, as well as metabolic changes of the surviving pups, effects that seem to be mediated by 
TGR5. ${ }^{28,29}$ Despite emerging studies on the possible mechanistic function of BAs in animals, knowledge on BAs in human reproduction is scarce and difficult to obtain due to ethical concerns regarding research with gametes and embryos.

One of the strengths of this work is the study of two different types of human GCs: CGCs and MGCs. Moreover, to the best of our knowledge, we are the first to show the presence of the classic BA transporters NTCP and ASBT outside hepatocytes and ileocytes, respectively. However, the present study also has points that should be approached cautiously. First, it cannot be excluded that the freshly isolated GCs may have been contaminated with other types of cells (eg, vaginal epithelial cells, ovarian epithelial cells, ovarian stromal cells, theca cells) that may have affected the results of protein detection and thus would explain the discrepancies between the Western blot and immunohistochemistry results. For example, cancerous epithelial ovarian cells have previously been shown to express $A B C C 4 .{ }^{30}$ In GC cultures, however, the majority of cells stained positive for MISR-II, indicating that, at least in cultures, contamination with other cells was minimal. Second, the FF is naturally rich in steroid hormones. In the present study, gonadotropin-stimulating steroid hormone was not added to the culture medium, which may alter GC function. However, the steroidogenic capacity of the cultured cells was intact, indicating that the cultivated cells were vital.

In summary, the results of the present study suggest that BAs likely reach ovarian follicles by both passive and active transport from the blood compartment. Further studies are warranted to gain insight into the regulation of BA transport from blood into FF and into a potential impact of dysfunctional transport on reproductive physiology. Moreover, changes in blood BA composition possibly may impact FF BA composition, which would enable lifestyle and pharmacologic interventions to prevent and treat infertility.

\section{Acknowledgments}

We thank the staff of the Reproductive Medicine Laboratory of the University Medical Center Groningen for collection of patient materials, Tineke van der Sluis at the Laboratory of Pathology of the University Medical Center Groningen for expertise in immunohistochemistry, Martijn Koehorst for expert technical assistance in the bile acid measurements, Dr. Bruno Stieger (University Hospital Zurich, Zurich, Switzerland) for the NTCP antibody, and Prof. Paul A. Dawson (Wake Forest University School of Medicine, Winston-Salem, NC) for the ASBT antibody.

\section{Supplemental Data}

Supplemental material for this article can be found at http://doi.org/10.1016/j.ajpath.2019.06.011.

\section{References}

1. Siu MK, Cheng CY: The blood-follicle barrier (BFB) in disease and in ovarian function. Adv Exp Med Biol 2012, 763:186-192

2. Da Broi MG, Giorgi VSI, Wang F, Keefe DL, Albertini D, Navarro PA: Influence of follicular fluid and cumulus cells on oocyte quality: clinical implications. J Assist Reprod Genet 2018, 35: 735-751

3. Norris RP, Ratzan WJ, Freudzon M, Mehlmann LM, Krall J, Movsesian MA, Wang H, Ke H, Nikolaev VO, Jaffe LA: Cyclic GMP from the surrounding somatic cells regulates cyclic AMP and meiosis in the mouse oocyte. Development 2009, 136:1869-1878

4. Matzuk MM, Burns KH, Viveiros MM, Eppig JJ: Intercellular communication in the mammalian ovary: oocytes carry the conversation. Science 2002, 296:2178-2180

5. Sugiura K, Eppig JJ: Society for reproductive biology founders' lecture 2005. Control of metabolic cooperativity between oocytes and their companion granulosa cells by mouse oocytes. Reprod Fertil Dev 2005, 17:667-674

6. Pangas SA, Matzuk MM: The art and artifact of GDF9 activity: cumulus expansion and the cumulus expansion-enabling factor. Biol Reprod 2005, 73:582-585

7. Wallace M, Cottell E, Gibney MJ, McAuliffe FM, Wingfield M, Brennan L: An investigation into the relationship between the metabolic profile of follicular fluid, oocyte developmental potential, and implantation outcome. Fertil Steril 2012, 97. 1078,84.e1-8

8. Nagy RA, van Montfoort AP, Dikkers A, van Echten-Arends J, Homminga I, Land JA, Hoek A, Tietge UJ: Presence of bile acids in human follicular fluid and their relation with embryo development in modified natural cycle IVF. Hum Reprod 2015, 30:1102-1109

9. Stouffer RL, Xu F, Duffy DM: Molecular control of ovulation and luteinization in the primate follicle. Front Biosci 2007, 12: 297-307

10. Zhou H, Ohno N, Terada N, Saitoh S, Fujii Y, Ohno S: Involvement of follicular basement membrane and vascular endothelium in blood follicle barrier formation of mice revealed by 'in vivo cryotechnique'. Reproduction 2007, 134:307-317

11. Hillier SG, Whitelaw PF, Smyth CD: Follicular oestrogen synthesis: the 'two-cell, two-gonadotrophin' model revisited. Mol Cell Endocrinol 1994, 100:51-54

12. Gautier T, Becker S, Drouineaud V, Menetrier F, Sagot P, Nofer JR, von Otte S, Lagrost L, Masson D, Tietge UJ: Human luteinized granulosa cells secrete apoB100-containing lipoproteins. J Lipid Res 2010, 51:2245-2252

13. Vitek L, Haluzik M: The role of bile acids in metabolic regulation. J Endocrinol 2016, 228:R85-R96

14. Kuipers F, Stroeve JH, Caron S, Staels B: Bile acids, farnesoid X receptor, atherosclerosis and metabolic control. Curr Opin Lipidol 2007, 18:289-297

15. Ferdinandusse S, Houten SM: Peroxisomes and bile acid biosynthesis Biochim Biophys Acta 2006, 1763:1427-1440

16. Meier PJ, Stieger B: Bile salt transporters. Annu Rev Physiol 2002, 64 635-661

17. Smith LP, Nierstenhoefer M, Yoo SW, Penzias AS, Tobiasch E, Usheva A: The bile acid synthesis pathway is present and functional in the human ovary. PLoS One 2009, 4:e7333

18. Pelinck MJ, Vogel NE, Hoek A, Simons AH, Arts EG, Mochtar MH, Beemsterboer S, Hondelink MN, Heineman MJ: Cumulative pregnancy rates after three cycles of minimal stimulation IVF and results according to subfertility diagnosis: a multicentre cohort study. Hum Reprod 2006, 21:2375-2383

19. Pelinck MJ, Vogel NE, Hoek A, Arts EG, Simons AH, Heineman MJ: Minimal stimulation IVF with late follicular phase administration of the GnRH antagonist cetrorelix and concomitant substitution with recombinant FSH: a pilot study. Hum Reprod 2005, 20:642-648 
20. Groen H, Tonch N, Simons AH, van der Veen F, Hoek A, Land JA: Modified natural cycle versus controlled ovarian hyperstimulation IVF: a cost-effectiveness evaluation of three simulated treatment scenarios. Hum Reprod 2013, 28:3236-3246

21. Christian WV, Li N, Hinkle PM, Ballatori N: Beta-subunit of the ostalpha-ostbeta organic solute transporter is required not only for heterodimerization and trafficking but also for function. J Biol Chem 2012, 287:21233-21243

22. Ceryak S, Bouscarel B, Fromm H: Comparative binding of bile acids to serum lipoproteins and albumin. J Lipid Res 1993, 34:1661-1674

23. Jaspard B, Fournier N, Vieitez G, Atger V, Barbaras R, Vieu C, Manent J, Chap H, Perret B, Collet X: Structural and functional comparison of HDL from homologous human plasma and follicular fluid. A model for extravascular fluid. Arterioscler Thromb Vasc Biol 1997, 17:1605-1613

24. van Montfoort AP, Plosch T, Hoek A, Tietge UJ: Impact of maternal cholesterol metabolism on ovarian follicle development and fertility. J Reprod Immunol 2014, 104-105:32-36

25. Zhang JY, Diao YF, Oqani RK, Han RX, Jin DI: Effect of endoplasmic reticulum stress on porcine oocyte maturation and parthenogenetic embryonic development in vitro. Biol Reprod 2012, 86:128
26. Lin T, Diao YF, Kang JW, Lee JE, Kim DK, Jin DI: Tauroursodeoxycholic acid improves the implantation and live-birth rates of mouse embryos. Reprod Biol 2015, 15:101-105

27. Kim JS, Song BS, Lee KS, Kim DH, Kim SU, Choo YK, Chang KT, Koo DB: Tauroursodeoxycholic acid enhances the pre-implantation embryo development by reducing apoptosis in pigs. Reprod Domest Anim 2012, 47:791-798

28. Baptissart M, Vega A, Martinot E, Pommier AJ, Houten SM, Marceau G, de Haze A, Baron S, Schoonjans K, Lobaccaro JM, Volle DH: Bile acids alter male fertility through G-protein-coupled bile acid receptor 1 signaling pathways in mice. Hepatology 2014, 60: $1054-1065$

29. Baptissart M, Sedes L, Holota H, Thirouard L, Martinot E, de Haze A, Rouaisnel B, Caira F, Beaudoin C, Volle DH: Multigenerational impacts of bile exposure are mediated by TGR5 signaling pathways. Sci Rep 2018, 8:16875

30. Bagnoli M, Beretta GL, Gatti L, Pilotti S, Alberti P, Tarantino E, Barbareschi M, Canevari S, Mezzanzanica D, Perego P: Clinicopathological impact of ABCC1/MRP1 and ABCC4/MRP4 in epithelial ovarian carcinoma. Biomed Res Int 2013, 2013: 143202 